\title{
Monomelic Amyotrophy (Hirayama Disease) With Upper Motor Neuron Signs: A Case Report
}

\author{
Seung Don Yoo, MD, Hee-Sang Kim, MD, Dong Hwan Yun, MD, Dong Hwan Kim, MD, \\ Jinmann Chon, MD, Seung Ah Lee, MD, Sung Yong Lee, MD, Yoo Jin Han, MD
}

Department of Physical Medicine and Rehabilitation, Kyung Hee University School of Medicine, Seoul, Korea

\begin{abstract}
Monomelic amyotrophy (MMA), also known as Hirayama disease, is a sporadic juvenile muscular atrophy in the distal upper extremities. This disorder rarely involves proximal upper extremities and presents minimal sensory symptoms with no upper motor neuron (UMN) signs. It is caused by anterior displacement of the posterior dural sac and compression of the cervical cord during neck flexion. An 18-year-old boy visited our clinic with a 5 -year history of left upper extremity pain and slowly progressive weakness affecting the left shoulder. Atrophy was present in the left supraspinatus and infraspinatus. On neurological examination, positive UMN signs were evident in both upper and lower extremities. Electrodiagnostic study showed root lesion involving the fifth to seventh cervical segment of the cord with chronic and ongoing denervation in the fifth and sixth cervical segment innervated muscles. Cervical magnetic resonance imaging (MRI) showed asymmetric cord atrophy apparent in the left side and intramedullary high signal intensity along the fourth to sixth cervical vertebral levels. With neck flexion, cervical MRI revealed anterior displacement of posterior dural sac, which results in the cord compression of those segments. The mechanisms of myelopathy in our patient seem to be same as that of MMA. We report a MMA patient involving proximal limb with UMN signs in biomechanical concerns and discuss clinical importance of cervical MRI with neck flexion. The case highlights that clinical variation might cause misdiagnosis.
\end{abstract}

Keywords Monomelic amyotrophy, Pyramidal signs, Biological assays

\section{INTRODUCTION}

Monomelic amyotrophy (MMA), also known as Hiraya-

Received April 30, 2013; Accepted December 18, 2013

Corresponding author: Sung Yong Lee

Department of Physical Medicine and Rehabilitation, Kyung Hee University School of Medicine, 26 Kyungheedae-ro, Dongdaemun-gu, Seoul 130-701, Korea

Tel: +82-2-440-7246, Fax: +82-2-440-7260, E-mail: stylistlsy@gmail.com

(c) This is an open-access article distributed under the terms of the Creative Commons Attribution Non-Commercial License (http://creativecommons. org/licenses/by-nc/3.0) which permits unrestricted noncommercial use distribution, and reproduction in any medium, provided the original work is properly cited.

Copyright $\odot 2015$ by Korean Academy of Rehabilitation Medicine ma disease, is a sporadic juvenile muscular atrophy in the distal upper extremities, which predominantly affects the lower cervical cord (e.g., seventh and eighth cervical vertebra levels). This disorder usually develops in the late teens and early twenties with a male preponderance. Clinical features of MMA include insidious onset and slow progression. MMA typically exhibits unilateral or asymmetric weakness and atrophy of the distal upper extremity. But sensory disturbance, reflex change and upper motor neuron (UMN) signs are rare.

Although several case reports have described patients with MMA presenting typical manifestations including distal limb involvement, there has been no description 
of MMA presenting with proximal limb involvement with positive UMN sign. We report an atypical MMA patient with proximal upper extremity involvement and positive UMN signs in biomechanical concerns ascertained by measuring cervical range of motion, especially flexion, in radiographs of the cervical spine [1].

\section{CASE REPORT}

An 18-year-old boy visited our clinic with a 5-year history of left upper extremity pain and slowly progressive weakness affecting left shoulder. Pain worsened when exposed to cold. He specifically noted difficulty in swimming and lifting heavy loads. He had also felt intermittent tremor and fasciculations in the left hand for the past 2 years. His past medical history was non-contributory, and he had no allergic disease. None of his family members had similar symptoms.

Tenderness at left deltoid muscle and atrophy in left supraspinatus and infraspinatus were evident. Neurological examination revealed positive Hoffmann signs, unsustained ankle clonus and strong, deep tendon reflex in

Table 1. Needle electrodiagnostic study

\begin{tabular}{|c|c|c|c|c|c|c|}
\hline & \multicolumn{3}{|c|}{ Spontaneous activity } & \multicolumn{3}{|c|}{ Motor unit action potential } \\
\hline & IA & Fib & PSW & $\operatorname{Amp}(\mathbf{m V})$ & Duration & Recruitment pattern \\
\hline Left SS & $\mathrm{N}$ & $(-)$ & $(-)$ & 6 & $\mathrm{~N}$ & Reduced \\
\hline Left IS & $\mathrm{N}$ & $(-)$ & $(-)$ & 7 & $\mathrm{~N}$ & Reduced \\
\hline Left deltoid & $\mathrm{N}$ & $(-)$ & $(-)$ & 7 & $\mathrm{~N}$ & Reduced \\
\hline Left triceps & $\mathrm{N}$ & $(-)$ & $(-)$ & 10 & $\mathrm{~N}$ & Reduced \\
\hline Left biceps & $\mathrm{N}$ & $(-)$ & $(-)$ & 6 & $\mathrm{~N}$ & Reduced \\
\hline Left FCR & $\mathrm{N}$ & $1+$ & $1+$ & $\mathrm{N}$ & $\mathrm{N}$ & Reduced \\
\hline Left ECR & $\mathrm{N}$ & $1+$ & $2+$ & 10 & $\mathrm{~N}$ & Reduced \\
\hline Left FCU & $\mathrm{N}$ & $(-)$ & $(-)$ & $\mathrm{N}$ & $\mathrm{N}$ & Complete \\
\hline Left supinator & $\mathrm{N}$ & $(-)$ & $(-)$ & 10 & $\mathrm{~N}$ & Reduced \\
\hline Left PT & $\mathrm{N}$ & $1+$ & $2+$ & 10 & $\mathrm{~N}$ & Reduced \\
\hline Left EDC & $\mathrm{N}$ & $(-)$ & $1+$ & 10 & $\mathrm{~N}$ & Reduced \\
\hline Left APB & $\mathrm{N}$ & $(-)$ & $(-)$ & $\mathrm{N}$ & $\mathrm{N}$ & Complete \\
\hline Left ADM & $\mathrm{N}$ & $(-)$ & $(-)$ & $\mathrm{N}$ & $\mathrm{N}$ & Complete \\
\hline Left TA & $\mathrm{N}$ & $(-)$ & $(-)$ & $\mathrm{N}$ & $\mathrm{N}$ & Complete \\
\hline Left PL & $\mathrm{N}$ & $(-)$ & $(-)$ & $\mathrm{N}$ & $\mathrm{N}$ & Complete \\
\hline Left VM & $\mathrm{N}$ & $(-)$ & $(-)$ & $\mathrm{N}$ & $\mathrm{N}$ & Complete \\
\hline Left cervical paraspinalis & $\mathrm{N}$ & $(-)$ & $(-)$ & $\mathrm{N}$ & $\mathrm{N}$ & Complete \\
\hline
\end{tabular}

IA, insertional activity; Fib, fibrillation potential; PSW, positive sharp wave; Amp, amplitude; SS, supraspinatus; IS, infraspinatus; FCR, flexor carpi radialis; FCU, flexor carpi ulnaris; ECR, extensor carpi radialis; PT, pronator teres; EDC, extensor digitorum communis; APB, abductor pollicis brevis; ADM, abductor digiti minimi; TA, tibialis anterior; PL, peroneus longus; VM, vastus medialis; $\mathrm{N}$, normal.

Table 2. Motor nerve conduction studies of both upper extremity

\begin{tabular}{|c|c|c|c|c|c|c|}
\hline \multirow{2}{*}{ Nerve } & \multicolumn{3}{|c|}{ Left side } & \multicolumn{3}{|c|}{ Right side } \\
\hline & Latency (ms) & Amplitude (mV) & $\mathrm{CV}(\mathrm{m} / \mathrm{s})$ & Latency (ms) & Amplitude (mV) & $\mathrm{CV}(\mathrm{m} / \mathrm{s})$ \\
\hline Median (APB) & 3.05 & 10.7 & 60.8 & 3.00 & 13.8 & 57.8 \\
\hline Ulnar (ADM) & 2.75 & 8.9 & 60.0 & 2.85 & 10.3 & 68.6 \\
\hline Radial (EIP) & 1.80 & 4.0 & 66.7 & 1.80 & 4.6 & 67.8 \\
\hline Axillary (deltoid) & 3.55 & 10.7 & - & 3.40 & 11.9 & - \\
\hline
\end{tabular}

$\mathrm{CV}$, conduction velocity; $\mathrm{APB}$, abductor pollicis brevis; $\mathrm{ADM}$, abductor digiti minimi; $\mathrm{EIP}$, extensor indicis proprius. 
Table 3. Sensory nerve conduction studies of both upper extremity

\begin{tabular}{lccccccc}
\hline \multirow{2}{*}{ Nerve } & \multicolumn{3}{c}{ Left side } & \multicolumn{3}{c}{ Right side } \\
\cline { 2 - 3 } \cline { 6 - 8 } & Latency (ms) & Amplitude $(\mu \mathbf{V})$ & $\mathbf{C V}(\mathbf{m} / \mathbf{s})$ & & Latency (ms) & Amplitude $(\mu \mathbf{V})$ & $\mathbf{C V}(\mathbf{m} / \mathbf{s})$ \\
\hline Median (index finger) & 3.00 & 79.7 & 46.7 & & 3.15 & 84.4 & 44.4 \\
Ulnar (little finger) & 3.30 & 27.2 & 42.4 & & 3.15 & 50.8 & 44.4 \\
Radial (thumb) & 3.30 & 26.5 & 42.4 & & 2.95 & 28.3 & 47.5 \\
\hline
\end{tabular}

$\mathrm{CV}$, conduction velocity.

Table 4. F-response tests on both upper extremity (unit, $\mathrm{ms}$ )

\begin{tabular}{lcc}
\hline \multicolumn{1}{c}{ Nerve } & Left side & Right side \\
\hline Median (APB) & 26.35 & 26.25 \\
\hline Ulnar (ADM) & 27.90 & 26.36 \\
CPN (EDB) & 48.80 & 48.60 \\
Tibial (AH) & 48.55 & 49.40 \\
\hline
\end{tabular}

$\mathrm{APB}$, abductor pollicis brevis; ADM, abductor digiti minimi; CPN, common peroneal nerve; EDB, extensor digitorum brevis; $\mathrm{AH}$, abductor halluces.

both upper and lower extremities. In physical examination, weakness in the left shoulder abduction on manual muscle testing (grade IV) was present. There was normal range of motion in left upper extremity and shoulder examination including empty can test. Neer and O'Brien tests showed no abnormal findings. Cranial nerve functions were normal and sensory disturbances were not found. Complete blood count, blood chemistry analysis, and immunoblot assay were not remarkable.

Needle electromyography (EMG) showed abnormal spontaneous activities at left flexor carpi radialis, extensor carpi radialis, pronator teres, extensor digitorum communis muscles and large amplitude of motor unit action potential with decreased interferencial patterns at left deltoid, supraspinatus, infraspinatus, triceps, biceps, extensor carpi radialis, supinator, pronator teres, and extensor digitorum communis muscles (Table 1). Motor nerve conduction study (NCS) for left median and ulnar nerves showed small amplitude compared to right side but latency and velocity were in the normal range (Table 2). Sensory NCS showed relatively small amplitude at the left ulnar nerve compared to the right side (Table 3). F-wave study showed slightly delayed latency at the left ulnar nerve compared to the right side (Table 4). These findings suggested root lesion involving the fifth to seventh cervical segment of the cord with chronic and ongo-

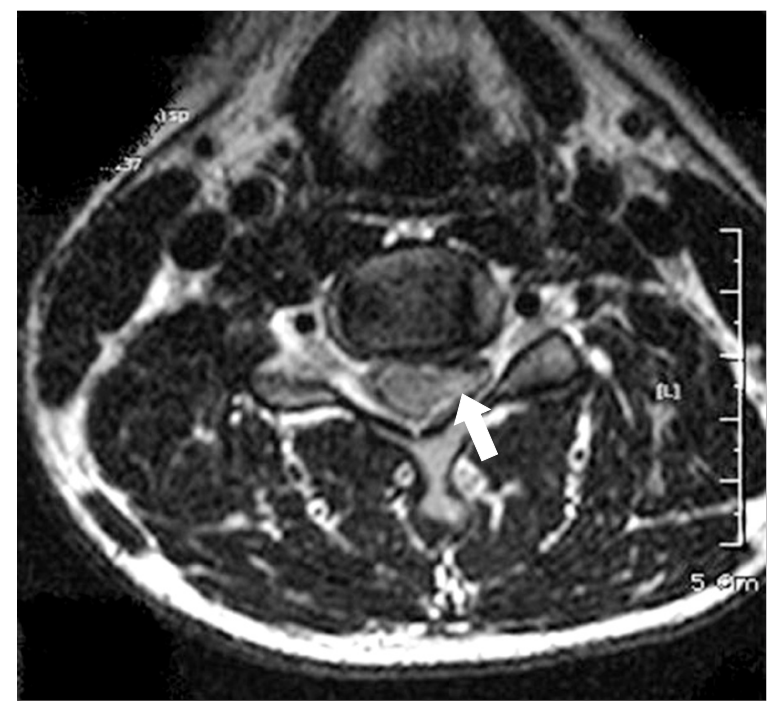

Fig. 1. Axial T2-weighted magnetic resonance imaging of the cervical spine at the C5-6 vertebral levels in neutral position. Spinal cord atrophy is apparent, especially on the left side (arrow).

ing denervation in the fifth and sixth cervical segment innervated muscles.

Cervical plain radiograph showed no significant change except kyphotic curvature. Two weeks later, cervical magnetic resonance imaging (MRI) revealed asymmetric cord atrophy apparent at the left side on T2-weighted axial images (Fig. 1). On T2-weighted sagittal images in the neutral position, intramedullary high signal intensity along the fourth to sixth cervical vertebral levels was shown without cervical cord compression (Fig. 2A). Further evaluation was needed to define the cause of the intramedullary high signal intensity along the fourth to sixth cervical vertebral levels. Cervical MRI was performed in the flexed neck position. T2-weighted sagittal images showed anterior displacement of posterior dural sac, resulting in the cervical cord compression of those levels (Fig. 2B). This MRI finding revealed cervical cord 

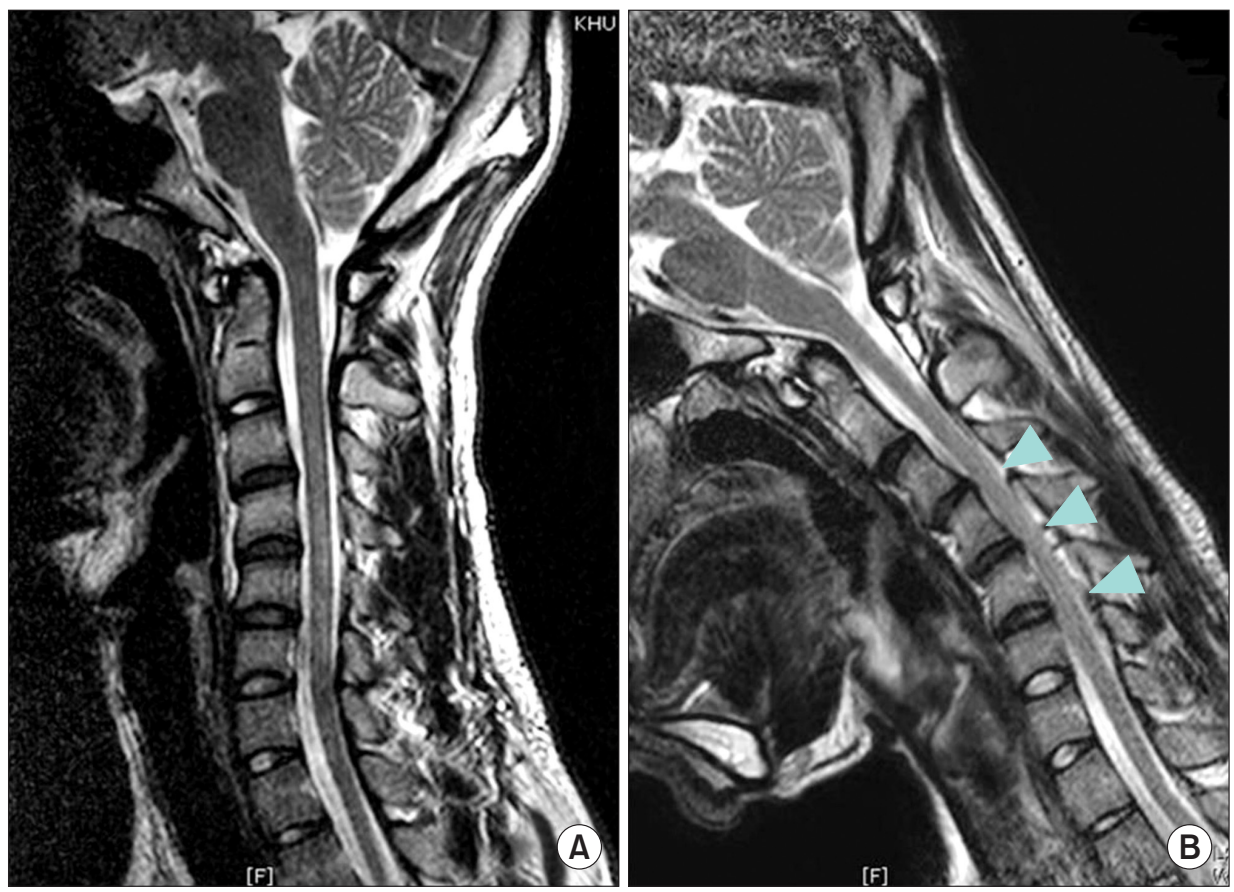

Fig. 2. (A) Midline sagittal T2weighted magnetic resonance imaging of the cervical spine in the neutral position reveals normal contour and intramedullary high signal intensity along the fourth to sixth cervical vertebral levels without cervical cord compression. (B) On sagittal T2-weighted images in the flexed neck position, there is anterior shifting of posterior dural sac below the third cervical vertebral level, compressing the spinal cord from third to sixth cervical vertebral levels (arrow).
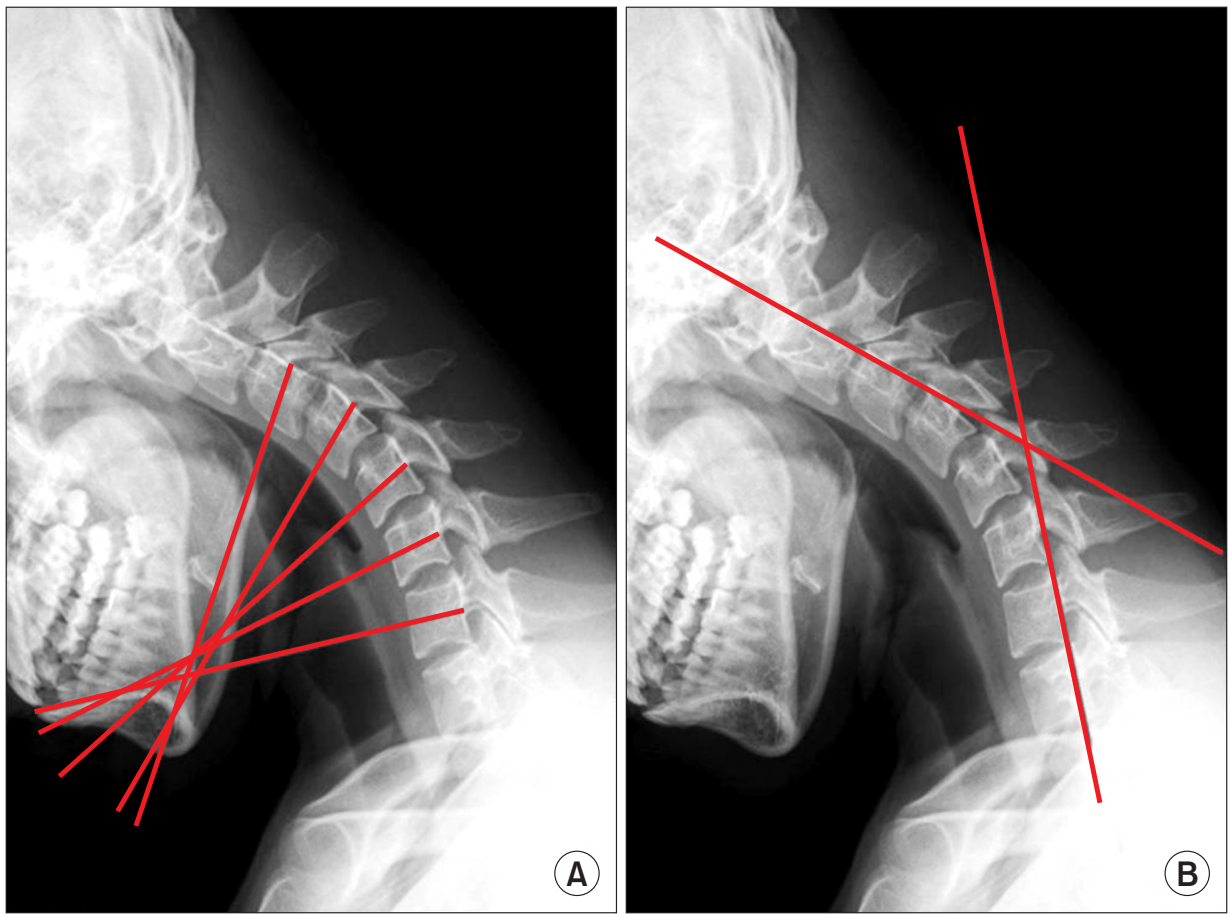

Fig. 3. (A) On lateral view of cervical X-ray in the flexed neck position. Inter-segmental angles were measured by drawing lines through the center of each vertebra body perpendicular to the long axis of each one. (B) Overall range of cervical flexed motion was calculated as the angle between tangent lines of the $\mathrm{C} 2$ vertebral body and the $\mathrm{C} 7$ vertebral body.

compression on neck flexion, which might have been the cause of myelopathy. These MRI findings strongly supported the diagnosis of MMA in our patient.

There were no abnormal findings from somatosensory evoked potentials, motor evoked potentials on both upper and lower extremities. Visual evoked potentials were also performed and there were no abnormal findings.

To examine cervical range of motion (CROM), both the segmental and overall range of cervical flexed motion (ROCFM) on radiographs were measured. Intersegmental angles were measured by drawing lines through the center of each vertebral body perpendicular to the long 
axis of each. Intersegmental angles from segment C3/C4 to $\mathrm{C} 6 / \mathrm{C} 7$ were $12.14^{\circ}, 15.83^{\circ}, 14.39^{\circ}$, and $15.83^{\circ}$, respectively (Fig. 3A). Overall ROCFM calculated as the angle between tangent lines drawn from the posterior aspects of the $\mathrm{C} 2$ vertebral body and the $\mathrm{C} 7$ vertebral body was $48.85^{\circ}$ (Fig. 3B). These findings also supported the diagnosis of MMA.

Since the initial study, the patient was treated with pain reliving medication. Six months later, his pain improved and he was weaned off the medication. After a 1.5-year follow-up, no further progression of neurological findings has been noticed.

\section{DISCUSSION}

MMA is a rare disease, which may present a diagnostic challenge. But it does have typical features. Tashiro et al. [2] recently outlined the requirements for diagnosis: 1) distal predominant muscle weakness and atrophy in forearm and hand, although limbs with no apparent symptoms of MMA can show abnormality by EMG; 2) almost always involves unilateral upper extremity; 3 ) onset between ages 10 to early 20s; 4) insidious onset with gradually progress for the first several years, followed by stabilization; 5) no lower extremity involvement; 6) no sensory disturbance and tendon reflex abnormalities; and 7) exclusion of other diseases (e.g., motor neuron disease, multifocal motor neuropathy, brachial plexopathy, spinal cord tumors, syringomyelia, cervical vertebral abnormalities, anterior interosseous or deep ulnar neuropathy).

Although diagnostic criteria for MMA proposed, several atypical cases were reported. Pradhan [3] reported cases with bilaterally symmetric limb involvement and Yilmaz et al. [4] reported MMA in a 7-year-old girl. Our patient had symptoms at left upper extremity and his age was late teens. So he met the diagnostic criteria for unilaterality and age, but had atypical characters including proximal limb involvement and positive UMN signs with strong deep tendon reflex. These clinical variations could make it difficult to diagnose MMA.

The diagnosis of MMA was on the basis of typical clinical manifestation until development of imaging study. Its causative mechanisms could be identified by cervical MRI in the flexed neck position. The pathogenic mecha- nism of MMA is attributed to forward displacement of the posterior lower cervical dural wall, resulting in asymmetric cervical cord flattening and cord compression when the neck is in flexion. Our patient had remarkable cervical cord compression in neck flexion although MRI in neutral position showed no evidence of cord compression. Therefore, the present case displayed characteristic MRI findings of dynamic cervical cord compression, emphasizing the clinical impotence of the cervical MRI in the flexed neck position for diagnosing MMA.

Regarding the pathomechanism of UMN signs, different distribution of stress in the cervical cord was suggested [5]. These authors reported that stress concentrated in mild cord compression is restricted to the gray matter; however, as cord compression increases, more stress becomes concentrated in both gray and white matter resulting in extensive cervical cord injury. Another case reports described that cervical flexion myelopathy patients with positive long tract sign feature gray and white matter lesions involving posterior horn [6]. So we could assume that our patient had a higher stress in cervical cord that could be a causative factor for positive UMN signs.

Another factor for extensive cord injury is hyperflexion of the neck. Xu et al. [1] reported differences in CROM between patients with Hirayama disease and healthy controls. In comparison of C3/4 to C6/7 intersegmental angles and ROCFM, the patients showed much greater degrees of angle than controls. The results of our patient were even greater than the mean degrees of the patients in all segments (Intersegmental angles: $7.28^{\circ}, 8.46^{\circ}$, $11.95^{\circ}, 10.88^{\circ}$ for mean degrees of patients, $12.14^{\circ}, 15.83^{\circ}$, $14.39^{\circ}, 15.83^{\circ}$ for our patient, respectively; ROCFM: $38.02^{\circ}$ for mean degrees of patients, $48.85^{\circ}$ for our patient, respectively). Therefore, this case suggest that hyperflexed CROM in a patient with MMA may serve as a causative factor UMN sign and that repeated neck flexion can lead to extensive cervical cord injury. This suggestion would be more conclusive if previous study on cervical segmental angles in Koreans had been done.

In conclusion, we report an atypical case of MMA with proximal involvement and positive UMN signs. In such cases, it is important to have careful evaluation thoroughly with cervical MRI with flexed neck position, even if neutral-position MRI appears no cord compression. 


\section{CONFLICT OF INTEREST}

No potential conflict of interest relevant to this article was reported.

\section{REFERENCES}

1. Xu X, Han H, Gao H, Hou C, Fan D, Fu Y, et al. The increased range of cervical flexed motion detected by radiographs in Hirayama disease. Eur J Radiol 2011; 78:82-6.

2. Tashiro K, Kikuchi S, Itoyama Y, Tokumaru Y, Sobue G, Mukai E, et al. Nationwide survey of juvenile muscular atrophy of distal upper extremity (Hirayama dis- ease) in Japan. Amyotroph Lateral Scler 2006;7:38-45.

3. Pradhan S. Bilaterally symmetric form of Hirayama disease. Neurology 2009;72:2083-9.

4. Yilmaz O, Alemdaroglu I, Karaduman A, Haliloglu G, Topaloglu H. Benign monomelic amyotrophy in a 7year-old girl with proximal upper limb involvement: case report. Turk J Pediatr 2011;53:471-6.

5. Kato Y, Kataoka H, Ichihara K, Imajo Y, Kojima T, Kawano S, et al. Biomechanical study of cervical flexion myelopathy using a three-dimensional finite element method. J Neurosurg Spine 2008;8:436-41.

6. Fujimoto Y, Oka S, Tanaka N, Nishikawa K, Kawagoe H, Baba I. Pathophysiology and treatment for cervical flexion myelopathy. Eur Spine J 2002;11:276-85. 